\title{
A Modified ISM Estimation Algorithm for Direction of Arrival (DOA) of Wideband Coherent Signal
}

\author{
Zhai Lijie, Duan Haisheng \\ Weinan Normal University, School of Mathematics and Physics, Weinan, Shaanxi, 714000
}

Keywords: wideband coherent signal; Direction of Arrival (DOA); modified ISM algorithm

\begin{abstract}
In order to perform the direction of arrival (DOA) estimation of wideband coherent signals based on the basic ISM algorithm, a modified ISM algorithm is proposed which can effectively judge the wideband coherent signals by utilizing the modified MUSIC algorithm with the characteristics of smooth decorrelation and whose performance is better than the basic ISM algorithm.
\end{abstract}

\section{Introduction}

As all we know, most of the DOA estimation algorithms of array signal are proposed against the narrow-band signals. However, with the development of technologies such as communication and electronic countermeasure, the application of broadband signals is increasing in the fields of radar, sonar, communication, biomedicine, etc. [1], which requires more accurate estimation when it gets such high attention. So far, there are two main types of estimation methods of wideband signal super-resolution: the Maximum Likelihood Estimation Method(MLM) [2] and the Signal Subspace Method (SSM). And there are also two main types of classical wideband signal subspace algorithms: the Incoherent Signal Subspace Method (ISM) and the Coherent Signal Subspace Method (CSM).

ISM [3] was proposed by Wax. The ISM algorithm is a direct extension of the narrow-band processing algorithm whose main idea is decomposing the wideband signal into non-overlapped narrow-band signals, and then processing the narrow-band signal subspace of each narrowband separately to obtain an initial angle estimation [4]. Since the method only utilizes part of the information of the wideband signal in each frequency band, the coherent source cannot be estimated with low estimation performance.

By using the modified MUSIC algorithm with the characteristics of smooth decorrelation function, the ISM algorithm is extended to the processing of wideband coherent signals, that is the modified ISM algorithm whose performance is better than basic ISM algorithm is used to the DOA estimation of the wideband coherent signal. By simulating the algorithm, the broadband coherent signal can be effectively resolved.

\section{Description of Basic ISM Algorithm}

ISM is an early method for wideband DOA estimation, The basic idea of this algorithm is decomposing the wideband signal into narrowband components that frequency domains do not overlap, and the narrow-band signal DOA estimation algorithm being used for direct processing at each narrow-band component, then performing arithmetic average value of the spectral estimation results of the respective sub-bands. Finally the wide-band signal DOA estimation is obtained [5].

When performing DOA estimation of a wideband signal, it is necessary to convert the observation data of time domain to the frequency domain by Discrete Fourier Transform. First of all, the data collected within the observation time $T_{0}$ is divided into $K$ segments, and the time length of each segment is $T_{s}=T_{0} / K$. Then point $J$ DFT transform of each segment data is performed to obtain $\mathrm{K}$ groups of mutually unrelated narrow-band components, and $K$ herein is referred to frequency domain snapshot number. Thus snapshot datas of $K$ groups are obtained, marked as $\mathbf{X}_{k}\left(f_{j}\right), k=1,2, \ldots, K, j=1,2, \ldots, J$. The data covariance matrix in the frequency domain is 
obtained by using the group $K$ data snapshot, and by which the ISM algorithm estimates the incident angle of signal [6].

Thus the output covariance matrix at the frequency point $f_{j}$ can be obtained:

$$
\mathbf{R}_{\mathrm{x}}\left(f_{j}\right)=\frac{1}{K} \sum_{k=1}^{K} \mathbf{X}_{k}\left(f_{j}\right) \mathbf{X}_{k}^{H}\left(f_{j}\right) \quad 1 \leq j \leq J
$$

Then the eigenvalue of $\mathbf{R}_{\mathrm{x}}\left(f_{j}\right)$ in the above equation is decomposed and get:

$$
\mathbf{R}_{\mathrm{x}}\left(f_{j}\right)=\sum_{i=1}^{M} \lambda_{i}\left(f_{j}\right) u_{i}\left(f_{j}\right) u_{i}^{H}\left(f_{j}\right)
$$

In the formula, the eigenvalues $\lambda_{i}\left(f_{j}\right)$ are arranged in descending order, and the eigenvector corresponding to the eigenvalues $\lambda_{i}\left(f_{j}\right)>\sigma^{2} \quad i=1,2, \ldots, P$ is expressed as: $\mathbf{U}_{s}\left(f_{j}\right)=\left[u_{1}\left(f_{j}\right) u_{2}\left(f_{j}\right) \ldots u_{P}\left(f_{j}\right)\right]$. The subspace spanned by $\mathbf{U}_{s}\left(f_{j}\right)$ is a signal subspace, and the eigenvector corresponding to the eigenvalues $\lambda_{i}\left(f_{j}\right)=\sigma^{2}(i=P+1, \ldots, M)$ is expressed as: $\mathbf{E}_{n}\left(f_{j}\right)=\left[u_{P+1}\left(f_{j}\right) u_{P+2}\left(f_{j}\right) \ldots u_{M}\left(f_{j}\right)\right]$. The subspace spanned by $\mathbf{E}_{n}\left(f_{j}\right)$ is a noise subspace.

The spectral estimate of the arithmetic mean for the ISM algorithm can be expressed as:

$$
P_{\text {music }}(\theta)=\frac{1}{\frac{1}{J} \sum_{j=1}^{J}\left\|\mathbf{a}^{H}\left(f_{j}, \theta\right) \mathbf{E}_{n}\left(f_{j}\right)\right\|^{2}}
$$

\section{Description of Modified ISM Algorithm}

The ISM algorithm is decomposing the wideband signal into non-overlapped narrow bands, and directly performing traditional narrowband method such as the MUSIC method,etc. processing to each subband. DOA estimation of the wideband signal is achieved on average after the spectral estimation results are obtained. However, the ISM algorithm fails to the coherent sources. The ISM algorithm is extended to the processing of wideband coherent signals [7] by using modified MUSIC algorithm with characteristics of smooth decoherence function. Firstly, the conjugate data is rearranged according to the modified MUSIC algorithm principle, namely:

$$
\mathbf{Y}\left(f_{j}\right)=\mathbf{J M X}^{*}\left(f_{j}\right)
$$

In the above formula, $\mathbf{J M}$ is the anti-angle exchange unit matrix of $M \times M$, "**" represents the complex conjugate, and the covariance matrix of $\mathbf{Y}\left(f_{j}\right)$ is:

$$
\mathbf{R}_{\mathbf{y}}\left(f_{j}\right)=E\left[\mathbf{Y}\left(f_{j}\right) \mathbf{Y}^{H}\left(f_{j}\right)\right]=\mathbf{J M R}_{x}^{*}\left(f_{j}\right) \mathbf{J M}
$$

Where $\mathbf{R}_{\mathrm{x}}\left(f_{j}\right)$ is the covariance matrix defined by equation (2). The $\mathbf{R}_{\mathrm{x}}\left(f_{j}\right)$ and $\mathbf{R}_{\mathrm{y}}\left(f_{j}\right)$ are averaged to obtain a covariance matrix after conjugate rearrangement:

$$
\begin{aligned}
& \mathbf{R}\left(f_{j}\right)=\left[\mathbf{R}_{x}\left(f_{j}\right)+\mathbf{R}_{y}\left(f_{j}\right)\right] / 2 \\
& =\left\{\mathbf{A}\left(f_{j}\right) \mathbf{R}_{x}\left(f_{j}\right) \mathbf{A}^{H}\left(f_{j}\right)+\mathbf{J} \mathbf{M}\left[\mathbf{A}\left(f_{j}\right) \mathbf{R}_{x}\left(f_{j}\right) \mathbf{A}^{H}\left(f_{j}\right)\right]^{*} \mathbf{J} \mathbf{M}\right\} / 2+\sigma^{2}\left(f_{j}\right) \mathbf{I}
\end{aligned}
$$

It can be proved that the covariance matrices $\mathbf{R}_{x}\left(f_{j}\right)$ and $\mathbf{R}_{y}\left(f_{j}\right)$ have the same signal subspace and also have the same noise subspace. the new signal subspace $\mathbf{U}_{S}\left(f_{j}\right)=\left[u_{1}\left(f_{j}\right), u_{2}\left(f_{j}\right), \ldots u_{P}\left(f_{j}\right)\right]$ and the noise subspace $\mathbf{E}_{n}\left(f_{j}\right)=\left[u_{P+1}\left(f_{j}\right) u_{P+2}\left(f_{j}\right) \ldots u_{M}\left(f_{j}\right)\right]$ can be obtained by having eigenvalue decomposition of $\mathbf{R}\left(f_{j}\right)$, and then the spatial spectrum in all 
sub-bands is averaged. Finally, the spatial spectrum estimation of the modified ISM algorithm is obtained as follows:

$$
P_{\text {music }}(\theta)=\frac{1}{\frac{1}{J} \sum_{j=1}^{J}\left\|\mathbf{E}_{n}\left(f_{j}\right)^{H} \mathbf{a}\left(f_{j}, \theta\right)\right\|^{2}}
$$

It can be seen from the above analysis that the modified ISM algorithm actually utilizes the conjugate reconstruction process of the data matrix, which is essentially the case where the the length of sub-array is equal to array number in the spatial smoothing approach. The modified ISM algorithm is capable of handling the situation of coherent sources from both directions without loss of array aperture. In the case of incoherent sources, since $\mathbf{R}_{x}\left(f_{j}\right)$ or $\mathbf{R}_{y}\left(f_{j}\right)$ is estimated from finite snapshot data, the use of $\mathbf{R}\left(f_{j}\right)$ in equation (6) for DOA estimation has an average meaning, so the estimated performance can be improved.

\section{Analysis on Computer Simulation Performance of Two Algorithms}

Simulation conditions: In the simulation experiment, we take a uniform linear array composed of 8 array elements. The signal sources are two broadband Gaussian signals of same power with incident angles of $8^{\circ}$ and $15^{\circ}$ respectively. The center frequency of the signal is $f_{0}$, and the spacing between elements is half wavelength of the signal intermediate frequency(IF), that is $d=c / 2 f_{0}=\lambda / 2$. The sampling frequency is $f_{s}=2.4 f_{0}$. The relative bandwidth of the signal is $40 \%$, and the noise is Gaussian white noise with zero mean and not correlated with the signal. The signal to noise ratio is $10 \mathrm{~dB}$.

Analysis of simulation results: It can be seen from the above simulation diagrams that when the two signals are coherent signals, the ISM algorithm fails, but the modified ISM algorithm can still correctly distinguish the signal orientation. Therefore, we can know that the performance of the ISM algorithm estimation is better than the basic ISM algorithm.

ISM Algorithm

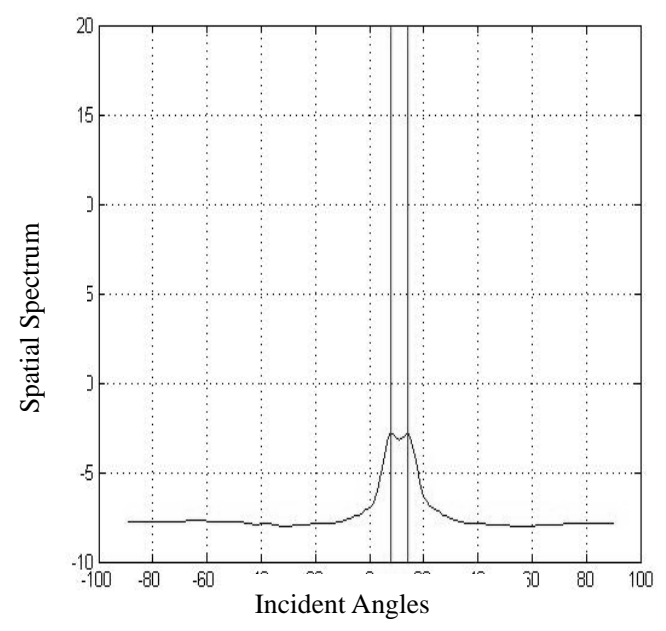

Figure 3 Spatial spectrum of the ISM algorithm under coherent sources 


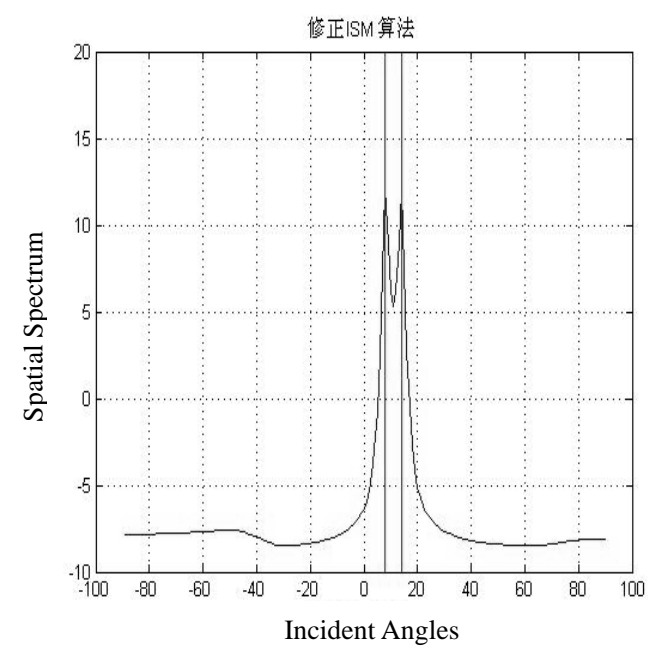

Figure 4 Spatial spectrum of the modified ISM algorithm under coherent sources

\section{Conclusion}

With the development of signal processing technology, wideband signals are more and more applied in signal processing systems, and people can obtain more information from broadband signals than from the narrowband. Therefore, DOA estimation of wideband signals is becoming more and more important.

This paper studies the DOA estimation of wideband signals. Firstly, the ISM method for DOA estimation of wideband incoherent signals is studied. The modified MUSIC algorithm based on data conjugate reconstruction is applied to the ISM algorithm, i.e. the modified ISM algorithm. Besides, the simulation experiment showed that the modified ISM algorithm can distinguish the coherent signal and improve the resolution ability.

\section{Acknowledgment}

Fund projects: Shaanxi Civil-Military Integration Project :( LDPC) Low density parity verification code in civil-military communications systems, Item Number: 16JMR12, University Level Project of Weinan Normal University: Research on the Application of X-ray Nondestructive Detection Technology in Agricultural Product Detection, Item Number: 16YKP008

\section{References}

[1] Li Feng. Research on Direction of Arrival (DOA) Estimation Algorithm for Smart Antenna [D]. Sichuan University, Chengdu,2009.6

[2] R. O. Schmidt. Multiple emitter location and signal parameter estimation [J]. IEEE Trans. AP, Mar. 1999, 34(3):276-280

[3] M. Wax, T. Shan, T. Kailath. Spatio-temporal spectral analysis by eigenstructure methods[C]. IEEE Trans. Acoust, Speech, Signal Processing, Aug. 1994, 32(8):817-827

[4] Wang Yongliang, Chen Hui et al. Spatial Spectrum Estimation Theory and Algorithms [M]. Beijing: Tsinghua University Press. 2004

[5] Bo Baolin. Study on DOA Estimation Algorithm of Wideband Array Signals [D]. Xidian University, Xi'an, 2007.6

[6] Li Feng. Research on Direction of Arrival (DOA) Estimation Algorithm for Smart Antenna [D]. Sichuan University, Chengdu,2009.6

[7] Wu Juan. Research on DOA Estimation Super-Resolution Algorithm of wideband signals [D]. Harbin Engineering University, Harbin, 2009.3 